\title{
Determining Alternative Train ODs under Cyclic Train Operation Mode for a Large-scale Rail Network
}

\author{
Yu-Lu TIAN" ${ }^{a}$, Lei NIE ${ }^{b}$, Hui-Ling FU ${ }^{c, *}$ and Tian-Qi LI ${ }^{d}$ \\ Beijing Jiaotong University, Shangyuancun No. 3, Haidian District, Beijing, China \\ a15120875@bjtu.edu.cn, 'Inie@bjtu.edu.cn, chlfu@bjtu.edu.cn, d15120839@bjtu.edu.cn \\ ${ }^{*}$ Corresponding author
}

Keywords: High-speed rail, Cyclic train operation mode, Line plan, Alternative train ODs.

\begin{abstract}
Although the cyclic train operation mode has been widely applied in many small-scale rail networks, it is more practical for a part of trains to use this mode under large-scale rail networks with complex structure and diverse travel demand. For a large-scale rail network, this paper analyzes how to determine alternative train ODs (origins and destinations). Specifically, we discuss the main elements, principles and methods of determination of cyclic and acyclic train ODs of the rail network. The proposed method is applied to a high-speed rail network in China to obtain alternative train ODs. The resulted scheme of train ODs can provide an important basis for designing a cyclic line plan.
\end{abstract}

\section{Introduction}

Cyclic mode is a train operation mode that each train line is the same among each period [8]. The mode has characteristics of high frequency, balanced-distributed departure time, and convenient transfer, thus it can optimize passenger service quality. Compared with the railway network in Europe and Japan, the railway network in China is larger and the travel demand is more complex. In this type of railway network, some of train ODs are suitable to be operated using cyclic mode, while the others are suggested to be operated still under acyclic mode.

Cyclic line plan is a basic plan of passenger railway operation, and it includes an important task of determining train ODs. Nevertheless, existing researches have not paid much attention to the alternative cyclic train ODs under cyclic mode. Oltrogge(1994) promoted a framework of determining the train lines. Bussieck et al. [1,2], Claessens [3], Scholl [4], Schöbel [5] and Borndörfer [6] studied on the line pool generation based on the framework. Bussieck [2] and Claessens [3] have taken cost optimization as a goal. Bussieck has adapted an outer approximation algorithm of DICOPT, and Claessens has used the branch and bound algorithm. Scholl [4] considered enumeration mothed to deal with customer-oriented line planning problem. Schöbel [5] and Borndörfer [6] formulated a model with objective of minimizing transfers. Schöbel considered to use Dantzig-Wolfe decomposition, and Borndörfer adapt shortest path algorithms. Jin-mei Li [7] has studied cyclic mode application on Beijing-Shanghai railway in China, although in reality China uses acyclic train operation mode,

This paper puts emphasis on generating alternative train ODs under cyclic mode combined with acyclic mode. Based on the recent passenger demand data and existing high-speed line plan, cyclic and acyclic train ODs generation principle and mothed will be analyzed and an adjustment mothed for some existing train ODs will be promoted.

\section{The Procedure of Determining the Train ODs}

The train OD is an important element in a line plan. The generation step of a cyclic line plan is shown in Fig. 1, in which the problem of determining the alternative train ODs is lacated. The problem is described as judging whether train OD pairs among terminals can be operated as cyclic or acyclic mode though a series of principles. Different from the optimization of an element in a line plan, this problem is suitable to be solved using operation knowledge rather than optimization methods. A framework is proposed for the determination of the problem, and the procedure is shown in Fig. 2. 


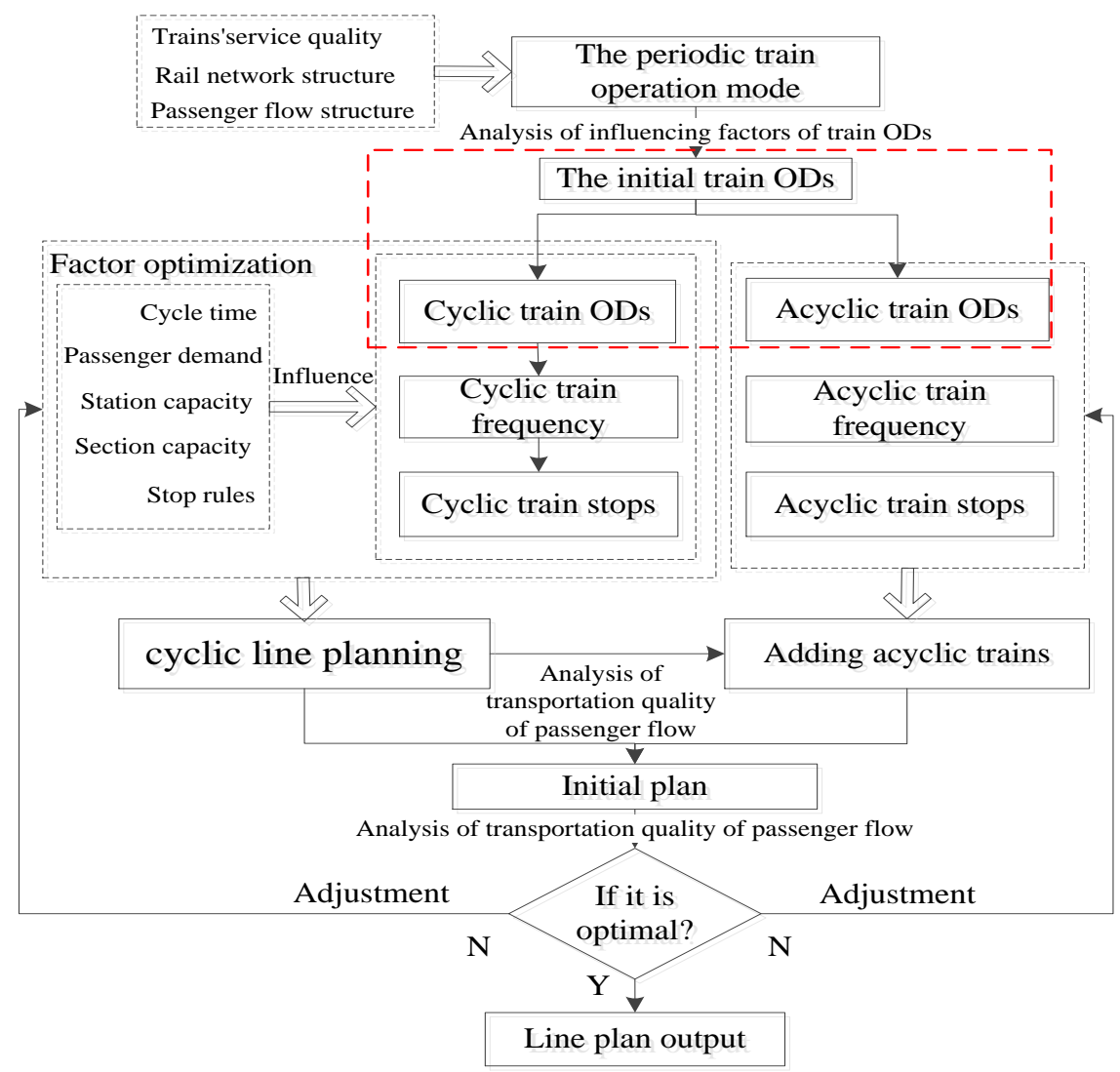

Fig. 1 Generation step of a cyclic line plan

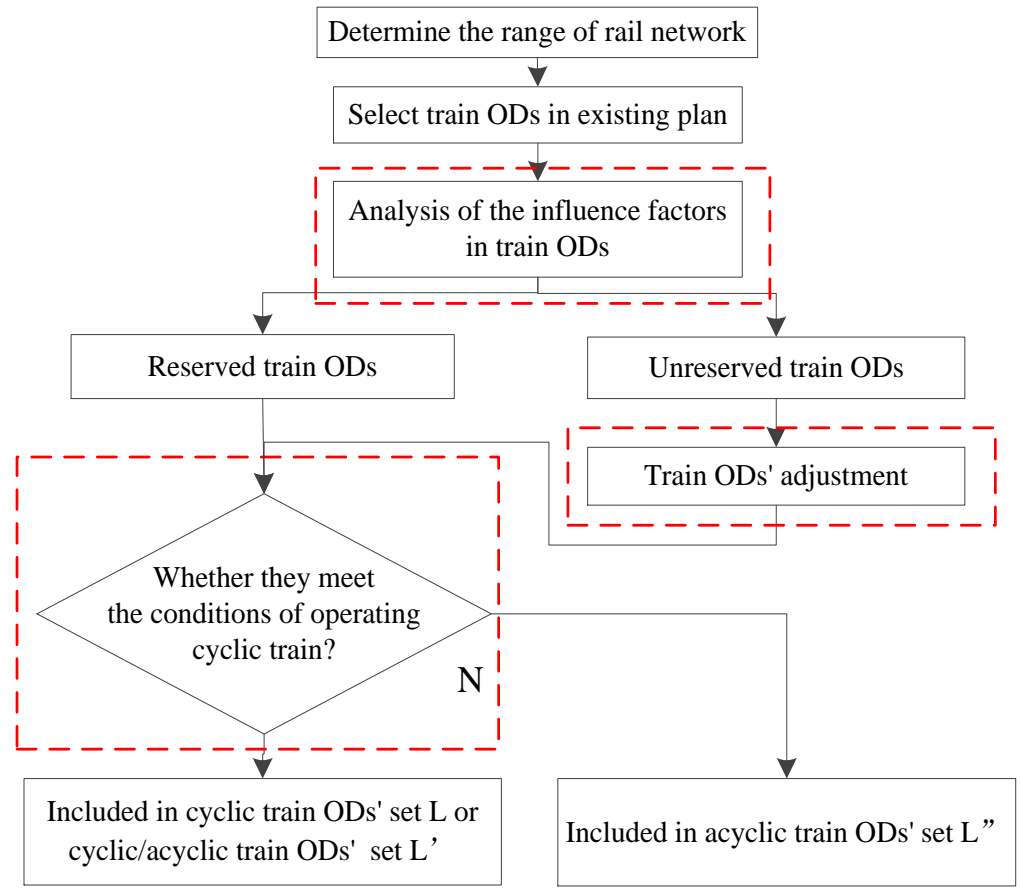

Fig. 2 Determination process of the alternative train ODs

Based on the existing train line plan, the alternative train ODs are divided into three sets through analyzing the influence factors, including: cyclic, acyclic and cyclic or acyclic alternative ODs, which provides the basis for line planning stage. Next we will analyze three sub-problems in detail which are indicated in Fig. 2 using dotted boxed, including: the influence factors in train ODs, cyclic train ODs' determination method and train ODs' adjustment. 


\section{Principle of Alternative Train ODs Generation}

\section{Determining Conditions of Train ODs}

When identifying alternative train ODs, the matching relation between trains and passenger flow in the existing plan should be fully considered. Taking the Chinese high-speed rail network as an example, there are particular characteristics of train ODs in the existing plan:

1. A large number of long-distance trains. The distance of the longest train has reached to $2,760 \mathrm{~km}$, but the ratio between long-distance passengers and long-distance train is not high.

2. Great variety of train ODs. The total kind of train ODs is more than 740 and the organization of train operation is complicated.

3. Unbalanced service quality. The train service frequencies of some passenger ODs are too low and the train service times are concentrated on a certain period of time.

Keeping these characteristics in mind, the paper analyzes the factors influencing train ODs and determines the train ODs in the existing plan that can be reserved or adjusted. The ratio between origin-destination passengers of a train and seating capacity of that train is a key factor which influences whether a train OD is to be reserved or not. The higher the ratio is, the higher the passenger load factor is in the train, and the better revenue of the train is.

Fig. 3 shows the analysis steps. The calculation process of the OD section passengers of a train OD is to divide each rail line in the network into several passenger-flow sections in accordance with the hubs connecting two or more rail lines, and to add all the quantities of passenger ODs of which origins are in the train OD's departure section and destinations are in the train OD's terminal section.

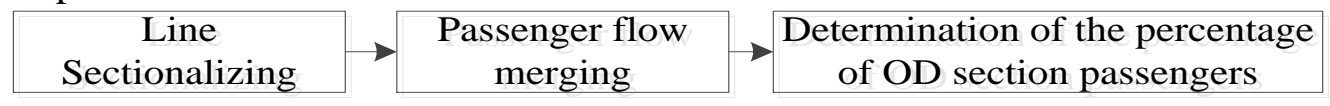

Fig. 3 Steps to determine the train ODs

The formula for calculating the the ratio between origin-destination passengers of a train and seating capacity of that train is stated as follows:

$$
\alpha=q_{O D} /\left(f_{O D} \times R\right) .
$$

Where, $\alpha$ represents the ratio-value, $q_{O D}$ represents the OD section passengers, $f_{O D}$ represents the train frequency, and $R$ represents the train seating capacity. Under the condition that the frequency is one at least, and the ratio-value should not be less than $\alpha_{0}$. Thus, the train OD of which origin-destination passengers are not less than $R \alpha_{0}$ can be reserved, or it should be adjusted. Because long-distance trains usually have to run through several sections and the OD section passengers are relatively fewer in long-distance OD, their $\alpha$-values are often lower than the $\alpha$-values of short-distance trains. Therefore, the value of $\alpha_{0}$ is associated with the running distance of the train OD.

\section{Determining Methods of Cyclic and Acyclic Train ODs}

The determination of cyclic and acyclic train ODs should consider station function, train running distance and the amount of passenger flow.

1. Station function

The station which can be used as the departure or arrival terminal is usually an important hub station. In order to facilitate the passenger transfer and the work organization of the stations, the terminal stations of a cyclic train OD should be hub stations with an EMU depot allocated near them.

2. Train running distance

The running distance of the cyclic trains should be within the range of dominant distance when the railway competes with other transport modes. The range of dominant distance of high-speed trains is almost 500-1,000km in Japan and European countries, but in China the upper bound of the range could reach about $1,500 \mathrm{~km}$. As the range is relatively fuzzy, a concept of membership function is introduced in determining the distance range of cyclic trains. Let $H(l)$ be the membership function of the fuzzy set 
"Dominant distance of high-speed trains", $H$ represents the fuzzy set "Dominant distance of high-speed trains", $l$ represents train running distance. The formula is shown in Eq. (2).

$$
H(l)=\left\{\begin{array}{lc}
1-\left(\frac{l_{1}-l}{l_{1}}\right)^{2} & 0<l<l_{1} \\
1 & l_{1} \leq l<l_{2} \\
1-\left(\frac{l-l_{2}}{l_{3}-l_{2}}\right)^{2} & l_{2} \leq l<l_{3} \\
0 & l \geq l_{3}
\end{array}\right.
$$

The values of $l_{1}, l_{2}, l_{3}$ depend on the distance distribution of the existing plan in 2017, which is shown in Table 1.

Table 1 The distance distribution of high-speed trains

\begin{tabular}{ccc}
\hline Distance distribution & Number of trains & Ratio \\
\hline $0-500$ & 823 & $38.32 \%$ \\
$500-1,000$ & 622 & $28.96 \%$ \\
$1,000-1,500$ & 466 & $21.69 \%$ \\
$1,500-$ & 237 & $11.03 \%$ \\
Total & 2,148 & $100.00 \%$ \\
\hline
\end{tabular}

The values of $l_{1}, l_{2}, l_{3}$ were 500, 1,000 and 1,500 in this paper. The train OD of which distance membership is greater than $H_{0}$ could be operated as cyclic mode, otherwise it will be operated as acyclic mode.

3. The amount of passenger flow

In order to increase the number of train ODs in each period and extend the service range of cyclic train ODs, the cycle time of the plan is set to be 2 hours, and the number of cycles in a day is 5 at least. In order to improve the load factor of cyclic trains, the $\alpha$-value should not be less than $\alpha$ ' in general and should be larger than $\alpha$ ". Thus, the origin-destination passengers of a cyclic train OD should reach $5 R \alpha$ '. For a cyclic or acyclic alternative train OD, it should be within a range of $R \alpha$ " to $5 R \alpha$ '. And for an acyclic train, it should be between $R \alpha_{0}$ and $5 R \alpha$ ". The values of $\alpha$ ' and $\alpha$ " are associated with the running distance of the train OD. The value of $\alpha_{0}$ is associated with the running distance of the train OD.

The determination procedure of the cyclic and acyclic train ODs is shown in Fig. 4.



Fig. 4 Determination procedure of the cyclic and acyclic train ODs

\section{Train OD Adjustment Strategies}

For those train ODs which are long-distance and do not meet the operational requirements in section 3.1, they should be truncated to two train ODs of which running distances are in the distance range of cyclic trains. The following strategies should be taken into consideration in selecting a transfer station of the train OD:

1. Arrange in large cities with developed economy and dense population.

2. Ensure balanced utilization of different transfer stations.

3. Consider to transfer using cyclic trains as much as possible. 
4. Train ODs with the same or a similar direction should be transferred at the same station.

The transfer stations of train ODs are usually chosen as those with the lowest percentage of transfer passengers. After the truncating treatment, the train ODs must be re-clustered according to the principles in section 3.2. The procedure for determining the transfer stations is shown in Fig. 5.

\begin{tabular}{|c|c|c|}
\hline $\begin{array}{l}\text { Find out the transfer } \\
\text { stations of the train OD }\end{array}$ & $\begin{array}{l}\text { Calculate the passenger } \\
\text { flow rate of each statior }\end{array}$ & \\
\hline
\end{tabular}

Fig. 5 Procedure for determining the transfer stations

\section{Case Analysis}

\section{Description of the Rail Network}

A sub high-speed rail network in China is selected to conduct a case study, as shown in Fig. 6.

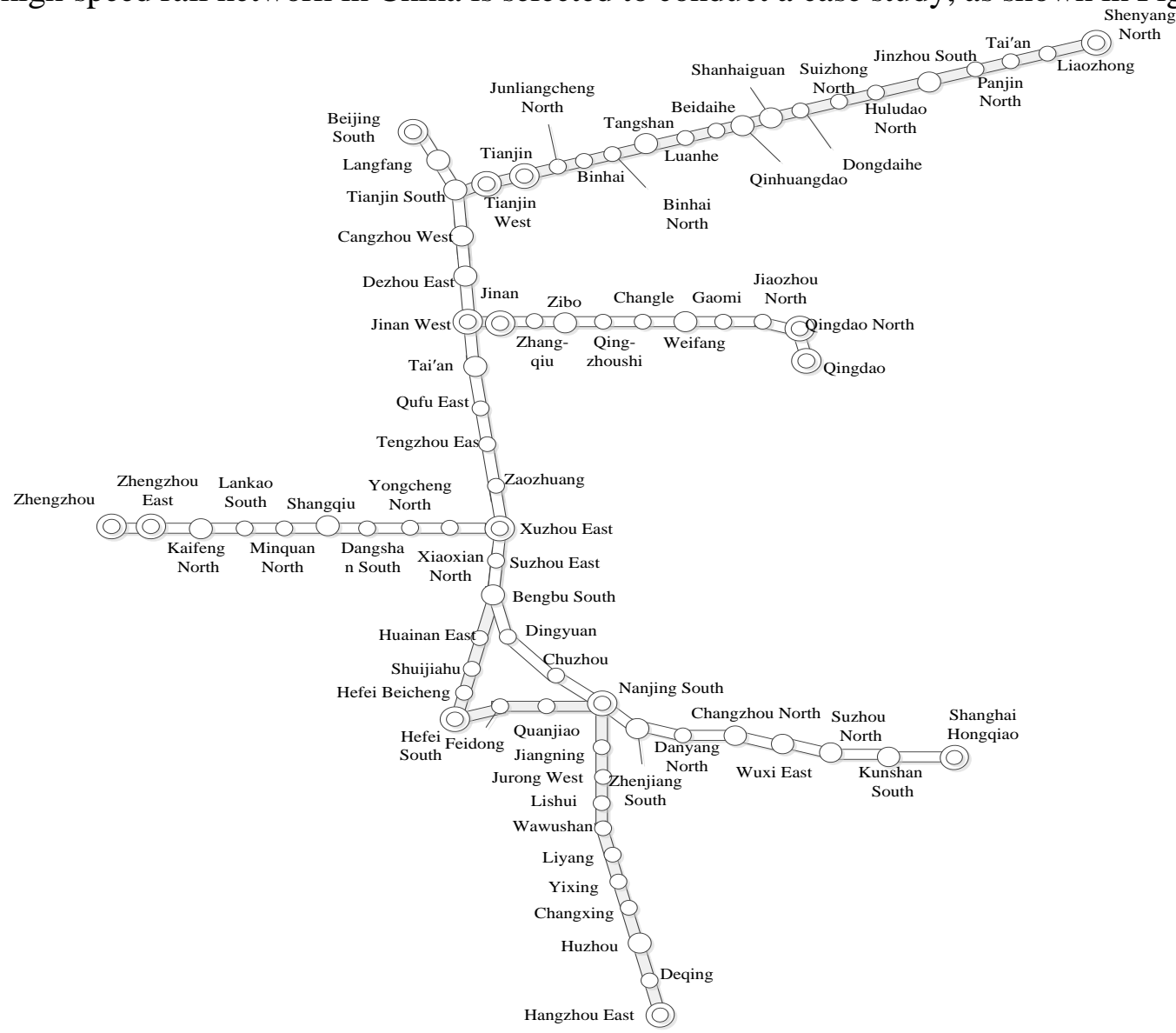

Fig. 6 A case study high-speed rail network

Based on the data of existing plan, all the stations and trains in the Beijing-Shanghai high-speed railway and connected lines are selected. The Beijing-Shanghai high-speed railway is divided into six passenger flow areas through Tianjin, Ji'nan, Xuzhou, Bengbu and Nanjing, while for other rail lines, each line is divided into one passenger flow area according to the connection and terminal station. In the rail network, Beijing, Shenyang, Tianjin, Ji'nan, Qingdao, Xuzhou, Hefei, Nanjing, Shanghai, Hangzhou and Hangzhou are hubs, and all of these stations have EMU depots nearby. They will be served as the terminal stations of cyclic trains.

\section{Determination of Train ODs}

A total of 38 train ODs in the network is selected from the ODs in the existing plan. According to the actual situation in China, the average train seating capacity $(R)$ is 1,000 , and the value of $H_{0}$ is set to be 0.3 in this case. The values of $\alpha_{0}, \alpha$ ' and $\alpha^{\prime \prime}$ are shown in Table 2. 
Table 2 Values of $\alpha_{0}, \alpha^{\prime}$ and $\alpha$ ”

\begin{tabular}{|c|c|c|c|c|}
\hline & $0<l \leq 500 \mathrm{~km}$ & $500 \mathrm{~km}<l \leq 1,000 \mathrm{~km}$ & $1,000 \mathrm{~km}<l \leq 1,500 \mathrm{~km}$ & $l>1,500 \mathrm{~km}$ \\
\hline$\alpha_{0}$ & $80 \%$ & $60 \%$ & $40 \%$ & $20 \%$ \\
\hline$\alpha^{\prime}$ & $80 \%$ & $60 \%$ & $40 \%$ & $/$ \\
\hline$\alpha^{\prime}$ & $40 \%$ & $30 \%$ & $20 \%$ & $/$ \\
\hline
\end{tabular}

According to the principles in section 3, the train ODs and the merged passengers are obtained, as shown in Table 3.

Table 3 Alternative train ODs

\begin{tabular}{|c|c|c|c|c|c|}
\hline No. & $\mathrm{O}$ & $\mathrm{D}$ & Distance $[\mathrm{km}]$ & OD passengers & Mode \\
\hline 1 & Beijing South & Shanghai Hongqiao & 1,318 & 13,807 & Cyclic \\
\hline 2 & Beijing South & Ji'nan West & 406 & 13,309 & Cyclic \\
\hline 3 & Beijing South & Nanjing South & 1,023 & 6,475 & Cyclic \\
\hline 4 & Beijing South & Xuzhou East & 692 & 6,557 & Cyclic \\
\hline 5 & Xuzhou East & Shanghai Hongqiao & 626 & 9,099 & Cyclic \\
\hline 6 & Ji'nan West & Shanghai Hongqiao & 912 & 5,814 & Cyclic \\
\hline 7 & Tianjin (West) & Shanghai Hongqiao & 1,213 & 3,977 & Cyclic \\
\hline 8 & Nanjing South & Shanghai Hongqiao & 295 & 43,145 & Cyclic \\
\hline 9 & Tianjin (West) & Shenyang North & 666 & 14,221 & Cyclic \\
\hline 10 & Ji’nan West & Qingdao & 413 & 24,229 & Cyclic \\
\hline 11 & Zhengzhou East & Xuzhou East & 360 & 8,538 & Cyclic \\
\hline 12 & Nanjing South & Hangzhou East & 256 & 20,089 & Cyclic \\
\hline 13 & Beijing South & Hangzhou East & 1,279 & 4,552 & Cyclic \\
\hline 14 & Beijing South & Qingdao & 819 & 8,329 & Cyclic \\
\hline 15 & Zhengzhou East & Shanghai Hongqiao & 986 & 4,341 & Cyclic \\
\hline 16 & Hefei South & Shanghai Hongqiao & 459 & 7,543 & Cyclic \\
\hline 1 & Beijing South & Qinghuangdao & 388 & 1,451 & Acyclic \\
\hline 2 & Beijing South & Tangshan & 241 & 1,105 & Acyclic \\
\hline 3 & Huainan East & Shanghai Hongqiao & 563 & 899 & Acyclic \\
\hline 4 & Ji’nan West & Zhengzhou East & 646 & 1,060 & Acyclic \\
\hline 5 & Zhengzhou East & Hangzhou East & 947 & 1,454 & Acyclic \\
\hline 6 & Qingdao & Zhengzhou East & 1,027 & 458 & Acyclic \\
\hline 7 & Qingdao & Hangzhou East & 1,269 & 560 & Acyclic \\
\hline 8 & Shenyang North & Shanghai Hongqiao & 1,964 & 738 & Acyclic \\
\hline 1 & Qingdao & Shanghai Hongqiao & 1,308 & 1,778 & Cyclic or Acyclic \\
\hline 2 & Beijing South & Hefei South & 1,000 & 2,750 & Cyclic or Acyclic \\
\hline 3 & Shenyang North & Ji'nan West & 972 & 2,088 & Cyclic or Acyclic \\
\hline 4 & Qingdao & Xuzhou East & 682 & 2,923 & Cyclic or Acyclic \\
\hline 5 & Hefei South & Hangzhou East & 413 & 2,759 & Cyclic or Acyclic \\
\hline
\end{tabular}

As shown in table 3, 29 rain ODs meet the determining requirements in the existing plan. According to the above principles, the alternative train ODs are decreased compared with the existing plan. Some train ODs like Bengbu South-Nanjing South which is short-distance and does not meet the operational requirements of cyclic trains is merged into the long-distance train ODs. Besides, a direct or transfer service can be provided for each OD within the network range theoretically. For cyclic and acyclic alternative train ODs, it can further use an optimization model to determine that it runs under a cyclic mode or an acyclic mode.

\section{Determination of Transfer Stations}

According to section 2, Beijing, Tianjin, Ji'nan, Xuzhou, Nanjing and Shanghai are selected as transfer hubs. Table 4 shows the train ODs that needed to be truncated in this case.

Table 4 Train ODs needed to be truncated

\begin{tabular}{ccccc}
\hline No. & O & D & Distance $[\mathrm{km}]$ & OD passengers \\
\hline 1 & Qingdao & Hefei & 990 & 252 \\
2 & Shenyang North & Qingdao & 1,370 & 383 \\
\hline
\end{tabular}


By using principles in section 3.3, the transfer stations of each train OD in the rail network are shown in Table 5.

Table 5 The transfer stations of each train OD

\begin{tabular}{|c|c|c|c|c|c|}
\hline O & D & $\begin{array}{c}\text { Transfer } \\
\text { stations }\end{array}$ & $\begin{array}{c}\text { Transfer } \\
\text { passengers }\end{array}$ & $\begin{array}{c}\text { Conveyed passengers } \\
\text { /Day }\end{array}$ & $\begin{array}{c}\text { The percentage of } \\
\text { transfer passengers }\end{array}$ \\
\hline Qingdao & Hefei South & Xuzhou & 232 & 1,442 & $16.09 \%$ \\
\hline Hefei South & Qingdao & Xuzhou & 192 & 934 & $20.56 \%$ \\
\hline Qingdao North & Shenyang North & Ji'nan & 302 & 1,610 & $18.76 \%$ \\
\hline Shenyang North & Qingdao North & Ji'nan & 229 & 1,275 & $17.96 \%$ \\
\hline
\end{tabular}

In Table 5, the percentages of transfer passengers are within the acceptable range, and all of the transfer passengers among these train ODs can use transfer service by riding cyclic trains. No new train OD is added after the above train ODs are truncated.

\section{Conclusion}

Considering a train operation mode which combines the cyclic mode with acyclic mode, this paper proposes the principle of generating alternative train ODs for a large-scale rail network. Influencing factors of the station function, train running distance and the amount of passenger flow are analyzed. The paper also analyzes the existing train ODs in a sub high-speed railway network in China, determines the cyclic and acyclic train ODs according to the proposed strategies, and truncates some long-distance trains at selected transfer nodes. This study paves a way for the generation of a cyclic line plan and timetable. Future research can be devoted to determine the elements of train service frequency and stopping pattern of a line plan.

\section{Acknowledgement}

This research is jointly supported by the National Natural Science Foundation of China (Grant No. U1434207 ,61703030), a project of the Science and Technology Department of the China Railway Corporation (Grant No. 2016X005-D) .

\section{References}

[1] Bussieck, M., Winter, T., Zimmermann, U.. Discrete optimization in public rail transport. TU Braunschweig. 1997.

[2] Bussieck M.Linder T.Lübbecke M. A fast algorithm for near cost optimal line plans, J. Mathematical Methods of Operations Research, 59(3), (2004) 205-220.

[3] Claessens, M.T., van Dijk, N.M., Zwaneveld, P.J., 1998. Cost optimal allocation of passenger lines. European Journal of Operational Research 110, 474-489.

[4] Scholl, S., 2005. Customer-oriented Line Planning [Dissertation], University of Kaiserslautern.

[5] Schöbel, A., Scholl, S.. Line planning with minimal transfers. In 5th Workshop on Algorithmic methods and Models for Optimization of Railways. Number 06901 in Dagstuhl Seminar Proceedings. 2006a.

[6] Borndörfer R, Neumann M. Models for Line Planning with Transfers, J. Linienplanung, 2010.

[7] Jin-mei Li. Research on Cyclic Train Line Planning for High-Speed Railways, D. Beijing Jiaotong University, 2015.

[8] Hui Chen, Hao Yang. Study on the Operation Scheme of PDL With Periodic Train Operation Diagram, J. Railway Transportation ans Economy, 28(12), (2006) 80-83. 